\title{
Atypia Suspicious for Malignancy
}

National Cancer Institute

\section{Source}

National Cancer Institute. Atypia Suspicious for Malignancy. NCI Thesaurus. Code C8350.

A microscopic finding indicating the presence of an atypical neoplastic cellular infiltrate with cytologic characteristics and/or architectural patterns that are not diagnostic, but raise the possibility of malignancy. 